\title{
Zoledronic Acid in Breast Cancer Patients with Bone Metastasis
}

\author{
I. Abdel Halim, E. El Sherbiny and W. El-Sadda
}

\author{
Clinical Oncology and Nuclear Medicine Department, Mansoura University, \\ Mansoura, Egypt
}

Background: Zoledronic acid is a nitrogen containing bisphosphonate that has been proven to reduce osteoporosis and cancer induced osteolysis. Zoledronic acid has been the treatment of choice for the prevention of skeletal complications of bone metastasis (Pain, Pathological fracture) in patients with breast cancer, which have significant impact on the quality of life of patients.

The aim of the study is to evaluate the efficacy and tolerability of zoledronic acid in improving pain score and quality of life in patients with bone metastasis secondary to breast cancer.

Materials \& Methods: 150 patients with bone metastasis and pathologically confirmed carcinoma of the breast with ECOG PS 0-2 and adequate renal function during the period between January 2007 and January 2009 were enrolled. Treatment consisted of zoledronic acid $4 \mathrm{mg}$ IV over 15 minutes repeated every 3-4 weeks concurrently with chemotherapy and/or hormonal therapy or radiotherapy if treatment was needed. Zoledronic acid was administrated continuously until worsening in the performance status, Progression of the disease or severe adverse events. Pain was evaluated by the present pain intensity from McGill Melzack (PP1) questionnaire, quality of life (QOL) was assessed with functional assessment of cancer therapy (FACT) questionnaire.

Results: The median age was 48 years (range: 30 -65 years) median PS was 1, extent of disease (metastasis): 60 patients (40\%) had only one bone metastatic site and 30 patients $(20 \%)$ had more than 3 bone metastatic sites. The average number of administrated cycles was 12 cycles (Range 6-50) and the average accumulated dose was $48 \mathrm{mg}$ (24-200), 75 patients (50\%) received treatment continuously for 2 years. The calcium and creatinine serum levels were assessed before each infusion. Five patients $(3.3 \%)$ developed hypocalcaemia (calcium $<8 \mathrm{mg} / \mathrm{dl}$ ) and 2 patients $(1.3 \%)$ experienced a significant increase in serum creatinine $(>3 \mathrm{mg} / \mathrm{dl}), 12$ patients $(8 \%)$ had an increase of pain after first infusion of Zoledronic acid. The rate of objective reduction in pain with zoledronic acid was $48 \%$. The improvement of quality of Life occurred in 75 patients $(50 \%)$.

Conclusion: Zoledronic acid is an effective, well tolerated and easily administrated bisphosphonate with evidence of activity in the treatment of bone metastasis from breast cancer. Zoledronic acid significantly improves quality of life and pain score.

Key words: breast cancer, bone metastasis, zoledronic acid

Corresponding Author: Wael El-Sadda_ E-mail: waelsadda@gmail.com

\section{INTRODUCTION}

Bone metastases are common in breast cancer, occurring in about $65 \%-75 \%$ of patients with metastasis, of whom bone is the sole site in about $25 \%{ }^{1}$. Complications include pain, vertebral compression, pathological fracture and hypercalcaemia. These complications are due not only to tumor associated bone invasion and destruction but also to osteolysis that is mediated by tumor associated osteoclast activating factors ${ }^{2}$. Patients with bone metastasis are at high risk for potentially debilitating skeletal related events (SREs) that can substantially reduce their quality of life $\mathrm{e}^{3}$. The SREs associated with bone metastasis include: pathologic fracture, spinal cord compression, radiation therapy or surgery to bone, or change in the antineoplastic therapy to treat bone pain ${ }^{4,5}$. In addition to acute morbidity, SREs can have longer term implication for patients ${ }^{6}$. Moreover, pathologic fractures are associated with reduced overall survival in patients with bone metastasis from breast cancer ${ }^{7}$. Bisphosphonates are widely used in the treatment of patients with bone metastasis secondary to solid or hematological malignancies ${ }^{8}$. 
Bisphosphonates are potent inhibitors of bone resorption that suppress both the activities of mature osteoclasts and the formation of new osteoclasts 9 . Zoledronic acid increases apoptosis of metastatic breast tumor cells in the bone and could therefore reduces metastatic tumor burden, 10 these results support the use of zoledronic acid to reduce the incidence of bone metastasis in breast cancer ${ }^{9}$.

Bisphosphonates reduce skeletal complications, bone pain and hypercalcaemia in patients with breast cancer and metastatic bone disease ${ }^{10}$.

First generation bisphosphonate including etidronate and clodronate are relatively weak inhibitors of osteoclast mediated bone resorption and require high intravenous or daily oral doses to achieve clinical benefit. These compounds preceded several generations of nitrogen containing bisphosphonate, including pamidronate, ibandronate and most recently, zoledronic acid. Nitrogen containing bisphosphonates are more potent and have demonstrated clinical activity in patients with bone metastasis when doses are infused at monthly intervals. Among these zoledronic acid, an imidazole containing bisophosphonate, has been shown to be the most potent bisphosphonate in vitro and in vivo assays of osteoclast mediated bone resorption ${ }^{11}$. Zoledronic acid has antitumor activity against a variety of tumor cell lines both in vivo and in animal models by inhibiting osteoclast activity and may also have direct antitumor effects through induction of apoptosis $^{12}$.

The earliest clinical application of bisphosphonates in cancer patients were for the control of hypercalcaemia of malignancy and for the treatment of osteolytic bone lesions in patients with multiple myeloma and breast cancer. Patients with advanced breast cancer frequently develop bone metastases and patients with osteolytic lesions are at particularly high risk of developing debilitating skeletal complications.

These complications include bone pain, requiring strong analgesic and palliative radiotherapy, pathologic fractures and spinal cord compression, which limit mobility and often require surgical intervention, and potentially life threatening hypercalcaemia. Because patients with bone metastases from breast cancer may live for several years following the diagnosis of bone involvement, these events can cause long term morbidity and poorer quality of life. Therefore, a treatment that can reduce the incidence of these skeletal complications has obvious benefit for the patients. Notably, an infusion of zoledronic acid once a month has been shown to significantly reduce the occurrence and to delay the onset of these types of skeletal complications in patients with breast cancer ${ }^{13}$.

Zoledronic acid has delayed the onset of these skeletal related events; however the use of this drug is associated with acute reactions and renal toxicity ${ }^{15}$.

\section{AIM OF THE STUDY}

The aim was to evaluate the efficacy and tolerability of zoledronic acid in improving pain scores and quality of life in patients with bone metastases secondary to breast cancer.

Primary end point: Response to treatment in term of pain reduction and quality of life improvement.

\section{Secondary end point: Toxicites.}

\section{PATIENTS AND METHODS}

This study included 150 patients with bone metastasis and pathologically confirmed carcinoma of the breast during the period between January 2007 and January 2009 with radiological evidence of metastatic disease.

Before the first study treatment, medical history was taken, physical examination and bone scan were performed while CT scan and/or bone survey (x-ray of thoracic and lumbar vertebrae, ribs and pelvis) were sometimes performed for further assessment. At baseline, pain was assessed using Present Pain Intensity (PPI) scale from the McGill-Melzak questionnaire and analgesic use was scored.

\section{- Eligibility criteria:}

- Patients between 18 and 65 years.

- $\quad$ Performance status 0-2.

- $\quad$ Life expectancy $>3$ months.

- Adequate renal function.

- Concurrent treatment with chemotherapy or hormonal therapy and radiotherapy if treatment was needed.

- Oral informed consent. 
- $\quad$ Corrected serum calcium $\geq 8.0 \mathrm{mg} / \mathrm{dL}$ and $\leq 11.5$ $\mathrm{mg} / \mathrm{dL}$, serum creatinine $\leq 1.5 \mathrm{mg} / \mathrm{dL}$, and total bilirubin $\leq 1.5 \mathrm{mg} / \mathrm{dL}$.

\section{- Exclusion criteria:}

Patients were not eligible if they had received prior treatment with a bisphosphonate within 12 months of enrollment, were pregnant or lactating, or had clinically symptomatic brain metastases.

\section{Treatment schedule:}

Zoledronic acid 4mg IV infusion over 15 minutes repeated every 3-4 weeks concurrently with chemotherapy, hormonal therapy or radiotherapy if treatment was needed. zoledronic acid drip rate was 133 drops/minute. Zoledronic acid was administered continuously until worsening in the performance status, progression of the disease or severe adverse events. All patients were given calcium $500 \mathrm{mg}$ and vitamin D 400 IU supplement daily. Serum creatinine was monitored before each zoledronic acid dose.

Pain was assessed by means of the Present Pain Intensity (PPI) scale from the McGill-Melzak ${ }^{16}$ questionnaire which uses verbal descriptors; scores can range from 0-5 with higher scores indicating greater pain.

Patients recorded their daily PPI score and analgesic use. A daily analgesic score was calculated by assigning a score of 4 for a standard dose of a narcotic analgesic (e.g. $10 \mathrm{mg}$ of morphine) and a score of 1 for a standard dose of a non narcotic analgesic.

The quality of life was assessed with the functional Assessment of Cancer Therapy-Breast (FACT-B) questionnaire; scores on this self administered questionnaire can range from $0-156$ with higher scores indicating a better quality of life ${ }^{16}$.

Evaluation of treatment response was done every cycle by reductions in pain and an improvement in the quality of life. Imaging studies to determine the extent of disease were performed every 3 cycles.

Patients with a PPI score of at least 2, an analgesic score of at least 10 or both (averaged over the previous week) at baseline were assessed for the pain response at three week intervals. A pain response was defined as a two point reduction in the PPI score from baseline without an increase in the analgesic score or as a reduction of at least $50 \%$ in the analgesic score without an increase in the PPI score, either of which was maintained for at least 3 weeks.

Pain progression was defined as an increase in the PPI sore of at least one point from the nadir, an increase from baseline of at least $25 \%$ in the analgesic score.

The quality of life was assessed with the FACT-B, Questionnaire at baseline, every three weeks during therapy and every month after the completion of therapy. Patients were defined as having a quality of life response if they had a 16 points improvement in their FACT-B score, as compared with baseline, on two measurements obtained at least three weeks apart. Adverse events were classified according to the World Health Organization critera ${ }^{18}$. Evaluation of safety and toxicity was done every cycle.

\section{RESULTS}

\section{Patient's characteristics}

This study included 150 patients who were diagnosed with bone metastasis and pathologically confirmed carcinoma of the breast during the period from January 2007 through January 2009 in Clinical Oncology and Nuclear Medicine Department, Mansoura University. Patient's characteristics are presented in (Table 1).

\section{Response to treatment}

The rate of objective reduction in pain with zoledronic acid was 48\% (72 patients) after 6 infusions. Twelve patients $(8 \%)$ had an increase of pain after first infusion of zoledronic acid and this pain relieved in the following infusion. The improvement of quality of life occurred in 75 patients $(50 \%)$. (Table 2).

The most common adverse events (Table 3) associated with zoledronic acid were of grade $1 \& 2$. Five patients $(3.3 \%)$ developed hypocalcaemia $(<8 \mathrm{mg} / \mathrm{dl})$ and 2 patients $(1.3 \%)$ experienced an increase in serum creatinine $>3$ but $\leq 6 \times$ ULN (grade $3 \mathrm{AE}$ ).

Adverse events in 150 patients with bone metastases received zoledronic acid. (No G4 toxicity). 
Table 1: Patient characteristics.

\begin{tabular}{|c|c|c|}
\hline Number of patients & $n=150$ & \\
\hline Median age & 48 years & \\
\hline (range) & (30-65 years) & \\
\hline Performance status & No. of pts & $\%$ \\
\hline 0 & 50 & $33.3 \%$ \\
\hline 1 & 60 & $40 \%$ \\
\hline 2 & 40 & $26.7 \%$ \\
\hline \multicolumn{3}{|l|}{ Concurrent treatment } \\
\hline Radiotherapy & 120 & $80 \%$ \\
\hline Chemotherapy & 100 & $66.7 \%$ \\
\hline Hormonal TTT & 50 & $33.3 \%$ \\
\hline \multicolumn{3}{|c|}{ Number of bone metastatic sites } \\
\hline One site & 60 & $40 \%$ \\
\hline 2 sites & 40 & $26.7 \%$ \\
\hline 3 sites & 20 & $13.3 \%$ \\
\hline$>3$ sites & 30 & $20 \%$ \\
\hline
\end{tabular}

- $\quad$ The average number of administered zoledronic acid cycles were 12 (range 6-50).

The average accumulated dose was $48 \mathrm{mg}$ ( range $24-200 \mathrm{mg}$ ).

$50 \%$ of patients received zoledronic acid continuously for 2 years.

Table 2: Response to treatment.

\begin{tabular}{lcc}
\hline & No. of patients & \% \\
\hline Pain reduction & $72($ score 1$)$ & $48 \%$ \\
Quality of life & $75($ score $>100)$ & $50 \%$ \\
\hline
\end{tabular}

Table 3: Toxicity profile.

\begin{tabular}{lccc}
\hline$\neg$ & GI & G2 & G3 \\
\hline Bone pain & $50(33.3 \%)$ & $15(9.9 \%)$ & - \\
Nausea & $8(5.3 \%)$ & $4(2.7 \%)$ & - \\
\hline Fatigue & $6(4 \%)$ & $3(2 \%)$ & - \\
Fever & $9(6 \%)$ & $3(2 \%)$ & - \\
Vomiting & $8(5.3 \%)$ & $2(1.3 \%)$ & - \\
Myalgia & $5(3.3 \%)$ & $2(1.3 \%)$ & - \\
\hline Hypocalcaemia & $5(3.3 \%)$ & $5(3.3 \%)$ & $2(1.3 \%)$ \\
S. creatinine & $5(3.3 \%)$ & $4(2.7 \%)$ & - \\
\hline
\end{tabular}




Vol. 9 | No. 1-2 2013

\section{DISCUSSION}

Bone is a major metastatic site for breast cancer and the complications associated with bone metastases can result in significant skeletal morbidities, which are named as skeletal related events (SREs) and include severe bone pain, pathologic fracture, spinal cord compression and hypercalcaemia ${ }^{9}$. Bisphosphonate are inhibitors of bone resorption and are the current standard treatment option for preventing (SREs) associated with bone metastasis ${ }^{2}$. Zoledronic acid; a third generation bisphosphonate, has the strongest inhibitory activity against bone resorption and it is the only bisphosphonate that has demonstrated statistically significant reduction of SREs due to metastatic bone lesions from various cancers ${ }^{19}$. In addition zoledronic acid has also been demonstrated to exhibit direct antitumor effects, not only through the inhibition of proliferation and induction of apoptosis in cultured cancer cells, but also via interference with cancer cell adhesion to the bone matrix and inhibition of cell migration and invasion ${ }^{20}$. In this study, $33.3 \%$ of patients had more than 2 bony metastatic sites, zoledronic acid was administered every 3-4 weeks concurrently with chemotherapy (all patients received anthracycline as adjuvant chemotherapy and the first line treatment for metastatic disease was taxanes containing regimen). Hormonal treatment (either tamoxifen or aromatase inhibitor was prescribed for patients with positive ER and/or PR) plus zoledronic acid every 4 weeks. In the 120 patients received radiotherapy, zoledronic acid was given one week before radiotherapy and then every 3-4 weeks for 2 years. Radiotherapy was indicated for severe pain and weight bearing area with dose $2000 \mathrm{cGy} / 5$ fractions or $3000 \mathrm{cGy} / 10$ fractions) according to the guidelines in our center.

In our study, only one patient had impending pathological fracture in the humerus (as seen in plain $\mathrm{x}$-ray) which was improved with subsequent zoledronic acid injections and no spinal cord was recorded.

In this study, 12 patients (8\%) had an increase of pain after the first infusion of zoledronic acid, this result is similar to that reported by Costa and Mayor ${ }^{22}$ and after 6 infusion of zoledronic acid the rate of objective reduction in pain was $48 \%$ these results are comparable to that obtained by Machado et al. ${ }^{10}$ In this study, the improvement of quality of life occurred in 75 patients (50\%) 6 months after starting the treatment, these results are the same as that reported by Trinkaus et al. ${ }^{7}$ Most adverse events associated with zoledronic acid in this study were manageable and of G1 \& G2.
Zoledronic Acid in Breast Cancer Patients with Bone Metastasis

Because all IV bisphosphonates are associated with some degree of renal function impairment, serum creatinine was closely monitored before each infusion. It was reported that less than $10 \%$ of patients had elevated serum creatinine following infusion of Zoledronic acid ${ }^{4}$. In this study 2 patients $(1.3 \%)$ developed G3 elevation of serum creatinine these results are similar to that obtained by Rosen et $a l^{23}$.

\section{CONCLUSION}

Zoledronic acid is an effective well tolerated and easily administered bisphosphonate with evidence of activity in the treatment of bone metastasis from breast cancer. Zoledronic acid significantly improves the quality of life and pain score. It can be administered simultaneously with chemotherapy, hormonal therapy and radiotherapy without relevant clinical problems.

\section{REFERENCES}

1. Cameron D. Proven efficacy of zoledronic acid in the treatment of bone metastases in patients with breast cancer and other malignancies. Breast 2003;12(Suppl 2):S22-9.

2. Major PP, Cook R. Efficacy of bisphosphonates in the management of skeletal complications of bone metastases and selection of clinical endpoints. Am. J. Clin. Oncol. 2002 25;6 Suppl. 1(S10):S18.

3. Weinfurt KP, Li Y, Castel LD, Saad F, Timbie JW, Glendenning GA, et al. The significance of skeletalrelated events for the health-related quality of life of patients with metastatic prostate cancer. Ann. Oncol. 2005;16(4):579-84.

4. Rosen LS, Gordon D, Tchekmedyian NS, Yanagihara R, Hirsh V, Krzakowski M, et al. Long-term efficacy and safety of zoledronic acid in the treatment of skeletal metastases in patients with nonsmall cell lung carcinoma and other solid tumors: A randomized, Phase III, double-blind, placebo-controlled trial. Cancer 2004;100(12):2613-21.

5. Saad F, Gleason DM, Murray R, Tchekmedyian S, Venner P, Lacombe L, et al. Long-term efficacy of zoledronic acid for the prevention of skeletal complications in patients with metastatic hormone-refractory prostate cancer. Natl.Cancer Inst. 2004;96(11):879-82.

6. Saad F, Lipton A, Cook R, Chen YM, Smith M, Coleman R. Pathologic fractures correlate with reduced survival in patients with malignant bone disease. Cancer 2007;110(8):1860-7.

7. Trinkaus M, Simmons C, Myers. Skeletal Related Event (SREs) in Breast cancer Patients with bone metastases treated in monterial setting Supp Care Cancer. 2009. 
8. Pavlakis N, Schmidt R, Stockler M. Bisphosphonates for breast cancer. Cochrane Database Syst. Rev. 2005(3):CD003474

9. Jeong J, Lee KS, Choi YK, Oh YJ, Lee HD. Preventive effects of zoledronic acid on bone metastasis in mice injected with human breast cancer cells. J. Korean Med. Sci. 2011; 26(12):1569-75.

10. Machado M, Cruz LS, Tannus G, Fonseca M. Efficacy of clodronate, pamidronate and zoledronate in reducing morbidity and mortality in cancer patients with bone metastasis: A meta-analysis of randomized clinical trials. Clin.Ther. 2009; 31(5):962-79.

11. Theriault RL, Hortobagyi GN. The evolving role of bisphosphonates. Semin. Oncol. 2001;28(3):284-90.

12. Hirbe AC, Roelofs AJ, Floyd DH, Deng H, Becker SN, Lanigan LG, et al. The bisphosphonate zoledronic acid decreases tumor growth in bone in mice with defective osteoclasts. Bone 2009;44(5):908-16.

13. Hillner BE, Ingle JN, Berenson JR, Janjan NA, Albain KS, Lipton A, et al. American Society of Clinical Oncology guideline on the role of bisphosphonates in breast cancer. American Society of Clinical Oncology Bisphosphonates Expert Panel. J. Clin. Oncol. 2000;18(6):1378-91.

14. Berenson JRH, B.E., Kyle R A, Anderson K, Lipton A, Yee GC, Biermann JS. American Society of Clinical Oncology Clinical Practice Guidelines: The Role of Bisphosphonates in Multiple Myeloma. JCO 2002;20(11):3719-36.

15. Martin M. Denosumab superior to zoledronic acid for breast cancer patients with bone metastases. 2012. American Association for Cancer Research in The News.
16. Melzack R. The McGill Pain Questionnaire: Major properties and scoring methods. Pain 1975;1(3):277-99.

17. WHO. WHO handbook for reporting results of cancer treatment. 1997. World Health Organization, Geneva.

18. Yuasa T, Kimura S, Ashihara E, Habuchi $T$, Maekawa T. Zoledronic acid - a multiplicity of anticancer action. Curr.Med.Chem. 2007;14(20):2126-35

19. Ibrahim A, Scher N, Williams G, Sridhara R, Li N, Chen G, et al. Approval summary for zoledronic acid for treatment of multiple myeloma and cancer bone metastases. Clin. Cancer Res. 2003;9(7):2394-9.

20. Coleman RE, Major P, Lipton A, Brown JE, Lee KA, Smith M, et al. -Predictive value of bone resorption and formation markers in cancer patients with bone metastases receiving the bisphosphonate zoledronic acid. J.Clin. Oncol. 2005;23(22):4925-35.

21. Boissier S, Ferreras M, Peyruchaud O, Magnetto S, Ebetino FH, Colombel M, et al. Bisphosphonates inhibit breast and prostate carcinoma cell invasion, an early event in the formation of bone metastases. Cancer Res. 2000;60(11):2949-54.

22. Costa L, Major PP. Effect of bisphosphonates on pain and quality of life in patients with bone metastases. Nat.Clin. Pract.Oncol. 2009;6(3): 163-74.

23. Kohno N, Aogi K, Minami H, Nakamura S, Asaga T, Iino Y, et al. Zoledronic acid significantly reduces skeletal complications compared with placebo in Japanese women with bone metastases from breast cancer: A randomized, placebo-controlled trial. 2005 J. Clin. Oncol.;23(15):3314-21. 